\title{
Researching on AODV and PS-AODV Routing Protocols of Ad Hoc Network for Streaming Media
}

\author{
Tian Jie, Wang Yu, Lv Jianxing \\ Engineering college of CAPF \\ Xi'an, China 710086
}

\begin{abstract}
Streaming media on wireless Ad hoc network becomes increasing popular, because of its huge potential market demand. In this paper, we propose a PS-AODV routing protocol based on an algorithm for path selecting and acknowledge refused, make improvements on AODV. The result of simulation shows that the PS-AODV protocol improves packet delivery ratio, reduces average end-to-end latency and decreases routing overhead in transmission of streaming media.
\end{abstract}

Keywords-Ad Hoc, Routing Protocols, Media Streaming, NS2

\section{INTRODUCTION}

An ad hoc network is a set of wireless mobile nodes that form a dynamic autonomous network without the intervention of centralized access points or base stations. Different from traditional wireless networks, ad hoc networks don't need fixed network infrastructure and can be deployed as multi-hop packet networks rapidly and with relatively low price. Thus such networks can be very useful in the scenario where natural condition or time restraints makes it impossible to have infrastructure pre-deployed. The ad hoc networks can be used in emergency services, conference rooms, or even home and office devices. Mobile nodes in an ad hoc network have limited radio transmission range. To forward packets between Nodes that are unable to communicate directly intermediate nodes are used. Each node acts both as a router and as a host. The function of a routing protocol in MANET is to establish routes between different nodes. Much research has been done on routing in ad hoc network. The new generation wireless networks permit to have much higher transmission rate. IEEE $802.11 \mathrm{~b}$ supports up to 11 Mbps transmission rate, IEEE 802.11a can support even up to $54 \mathrm{Mbps}$. It therefore possible to run multimedia applications multimedia instant, messaging, environmental monitoring, distributed gaming over mobile ad hoc networks, video surveillance, mobile learning, file sharing, video conferencing and streaming.

MANET is composed of a set of wireless mobile nodes that form a dynamic autonomous network without the intervention of centralized access points or base station, which do not need fixed network infrastructure and can be deployed as networks rapidly. In MANET, each node acts both as a router and a host. Node transmission distance is limited, so if the distance between the source node and destination node is out of the transmission range, the communication between them must be forwarded through intermediate nodes. Node's transmission capacity and energy is limited, therefore the nodes at the center of the network topology will be far greater in load pressure and energy consumption than the other nodes, easily leading to network congestion. How to improve network reliability and control the congestion of network are important issues that Ad hoc networks need to address. The application of streaming media in both military and business is increasing, so it is more and more important to find out suitable routing protocols to support the QoS during the transmission of streaming media in MANET[1].

\section{Routing PROTOCOLS}

MANET routing protocols can be divided into proactive routing protocols and reactive routing protocols, also known as table-driven and on-demand-driven. Table-driven routing protocols require each node maintaining one or more routing information and updating it when network topology changes. As is to on- demand-driven routing protocols, routing discovery is carried out only when source node needs. Once it was established, and will be maintained until the destination node cannot be reached, or the path is no longer needed. Research [2] indicates that Compared with the proactive routing protocols, reactive routing protocol packet transmission delay larger, but less overhead, packet delivery rate, more suitable for mobile ad hoc networks. The classic protocols include Ad hoc On-Demand Distance Vector Routing (AODV)[3].

In Mobile Ad hoc NETworks (MANETs), the communication between nodes is done over wireless media without the use of wired base stations. Distant nodes communicate over multiple hops and nodes must cooperate with each other to provide routing and to avoid transmission problems. The challenges in ad hoc networks are attributed to mobility of intermediate nodes, absence of routing infrastructure, low bandwidth, and computational capacity of the nodes. The other challenge in ad hoc network is inefficient in energy consumption .The Quality of service (QoS) routing in MANETs is difficult because the network topology may change constantly. The design of a decentralized medium access control (MAC) protocol is another challenge for QoS in multimedia and real time application. Because the dynamic topology effects network performance and limited bandwidth, the design of QoS 
multicast routing protocols is complicated in comparison with traditional networks; so traffic load must be distributed to eliminate hot spots and provide load balancing in the network. QoS in MANETs is highly rely on routing and MAC layers; delivering packet from source to destination with service quality in MANETs is principally linked to the performance and characteristics of the dynamic routing protocol.

\section{A. AODV Routing Protocol}

AODV routing protocol use the hop-by-hop way forward packets. Routing table records the next hop to the destination node, and do not need to carry the complete routing information in packets. When the source node has not known route to destination node, broadcasting a RREQ route request packet to its neighbors. When a node receives RREQ in case it has routing information, it sends the reply RREP packet back to the destination. Otherwise, it rebroadcasts RREQ packet further to its neighbors. As the RREQ packet travels from the source to the destination it automatically sets up the reverse path from all nodes back to the source. In addition, each node maintains a purposes sequence number, When nodes need to establish the path to the destination node and received the RREQ to themselves, its value is automatically increased by 1 . The serial number included in the RREQ, RREP. When other nodes in the network receiving the control message(RREQ,RREP) included node path information, contrast this serial number and the serial number of the node routing in the local route cache in order to select fresher route and avoid the generation of loop. RREQ packet contains a TTL(Time To Live) to Avoid bringing the whole network routing requests broadcast. AODV protocol receiving the broadcast or HELLO messages from a new neighbor or failing to receive HELLO messages from a node that was previously in the neighborhood, indicates that the local connectivity has changed.

\section{B. Improved AODV Protocol}

The traditional AODV protocol has uneven nodes energy consumption, so there are some deficiencies in the energy balance. We make improvement in route discovery process. Node checks its load value when received a RREQ packet in forwarding RREQ packets process, if the node load is too high, it refuses to forward the RREQ packet until the load is reduced. In order to ensure network load balance, we introduce the metric values to measure the energy of nodes in the network. The link load conditions from source node to destination node depend on the highest load node. That is the link metric (m) value: $m=\max \left(m_{1}, m_{2}, m_{3} \cdots m_{n}\right)$ The energy of nodes located in the center of the network topology would be easily exhausted, because of maintaining a high load. We shall consider the available energy of node. The metric values of $i$ node in the link is: $m_{i}=b_{i} \times l_{i} . b_{i}$ is the percentage of usable energy of node. $l_{i}$ is the load value of node. $l_{i}=\frac{q_{i}}{c_{i}} q_{i}$ is the length of the waiting queue. $c_{i}$ is the maximum cache of node. We assume that all nodes are of the same size, that is $c_{i}=c$. Therefore, the metric value of the destination node will be: $m=\frac{\max \left(b_{1} l_{1}, b_{2} l_{2}, b_{3} l_{3} \cdots b_{n} l_{n}\right)}{c}$.We can remove $\mathrm{C}$ to simplify the calculation. So, $m=\max \left(b_{1} l_{1}, b_{2} l_{2}, b_{3} l_{3} \cdots b_{n} l_{n}\right)$. The destination node or the intermediate nodes with the route to destination node can make best option according to the metric values, if there are multiple paths to send the RREP packet. PS-AODV routing protocol add a field metric $(m)$ _in RREQ packets to record the maximum load of node when passed. When the source node initiate route request, the value of $\mathrm{m}$ is calculated first by using the method above and written the $m$ values in the RREQ packet before broadcasting. Meanwhile, we add a field $m_{t}$ in node routing table to record the value from the source node to the current node of $m$. After receiving the RREQ packet, the node calculated the value of $\mathrm{m}$ to measure the available energy of the current node. The node has three states as follow:

Paralysis: $m \geq a$;

Congestion: $b \leq m \leq a$;

Normal: $m<b$;

Where $\mathrm{a}, \mathrm{b}$ for the congestion factor.

Each node in the network has the right to decide to forward or discard the RREQ packet received according to its available energy state. When an intermediate node is in a "paralyzed" state, it will discard all received RREQ and no longer replied any route requests except it is the destination node of the link. When a node is in "congestion" state, it will reply route request existing in the routing table only, and won't forward any RREQ packets. When a node is in "normal" state, if it is the first time to receive the RREQ from the source node, the value in the RREQ will change the value of corresponding field $\mathrm{m}_{\mathrm{t}}$. Then, it compared the value of $m$ and the metric value of the node, the larger one is recorded in the RREQ packet before forwarding. If the node has received the RREQ from the same source node with the same ID, it will compare the value of $m$ with $m_{t}$, and discard the RREQ if the value of $m$ is smaller. Otherwise, it will update the value of $m_{t}$ in the routing table and sent node pointer which point the previous hop to the node that sent RREQ. In order to reduce load of network, RREQ is not forwarded. After receiving the first RREQ, the destination node may wait for some time to get more routes, then choose the route with the minimum value of $m$ and reply a RREP packet.

\section{Simulations AND Results}

In this article, we present results for the simulations performed using the NS2 environment. NS2 is simply an event-driven simulation tool that has proved useful in 
studying the dynamic nature of communication networks. Simulation of wireless networks and Protocols can be done using NS2.

\section{A. Simulation parameters}

The following is the performance comparison parameters of protocol:

$$
\text { packet deliveryratio }=\frac{\text { network throughput }}{\text { all the source nodes to send the packetbitsinunit time }}
$$

Average end-to-end delay

$=$ the time required for route discovery process

+packet queuing time in the cache

+link layer packet retransmission time

+propagation time;

routing overhead $=\frac{\text { all therouting controloverhead } \text { RREQRREP and RERR) }}{\text { destination nodereceiveddatapacket }}$
network survival time $=\frac{\text { the first noded eath time }+ \text { the } \frac{n}{2} \text { node death time }}{2}$

B. Simulation results

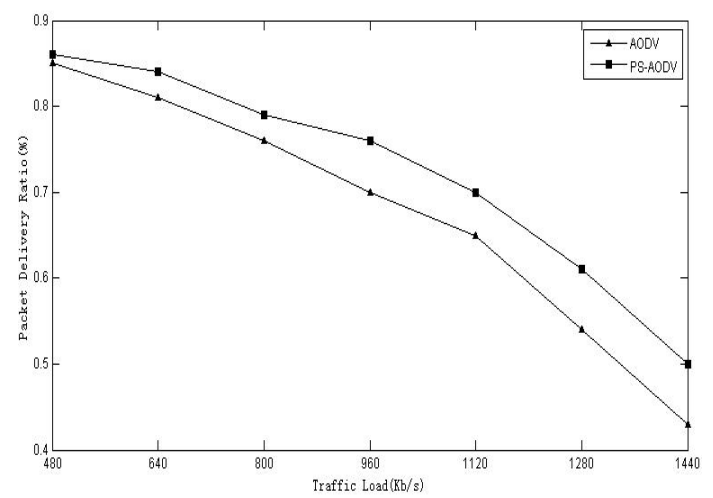

Figure 1. packet delivery ratio with different traffic load

Figure 1 shows that PS-AODV in packet delivery ratio have an advantage over AODV, especially when the network load increases. This is because AODV don't consider the load balance, leading to high packet loss and low packet delivery rate in high network load. The PS-AODV choose the path of lower load transfer data, and indirectly enhance the network throughput.

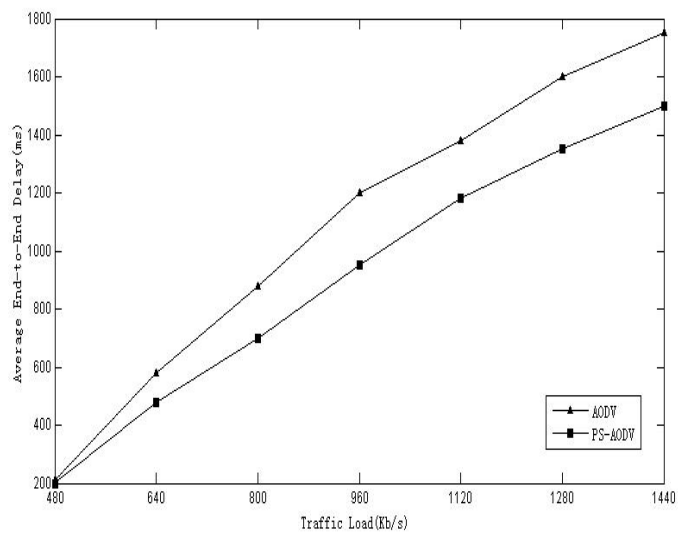

Figure 2. average end-to-end delay with different traffic load Figure 2 shows that the average end-to-end delay of
PS-AODV is lower than AODV, because factor of metric values includes the queue length, while the average end-to-end delay is mainly caused by the queuing time. PS-AODV chose a route of smaller queuing time, thereby reducing the network delay.

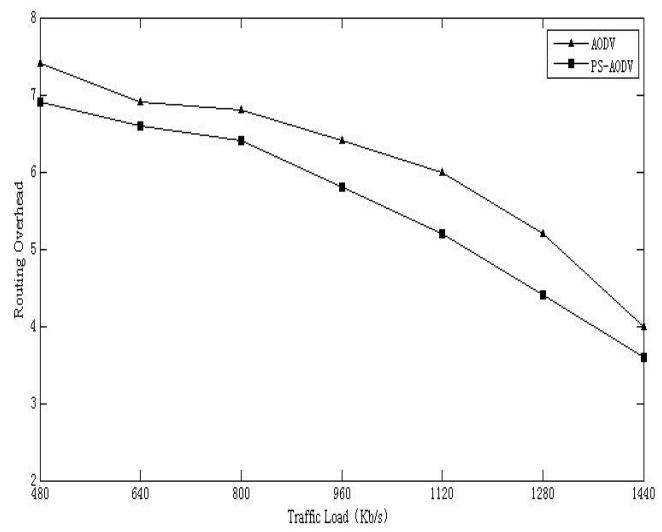

Figure 3. routing overhead with different traffic load

Figure 3 shows that PS-AODV's route overhead is lower than AODV. Because constantly forwarded RREQ packets accounts for a significant proportion in AODV route packets. While PS-AODV nodes drop RREQ packets according to the network load to reduce overhead.

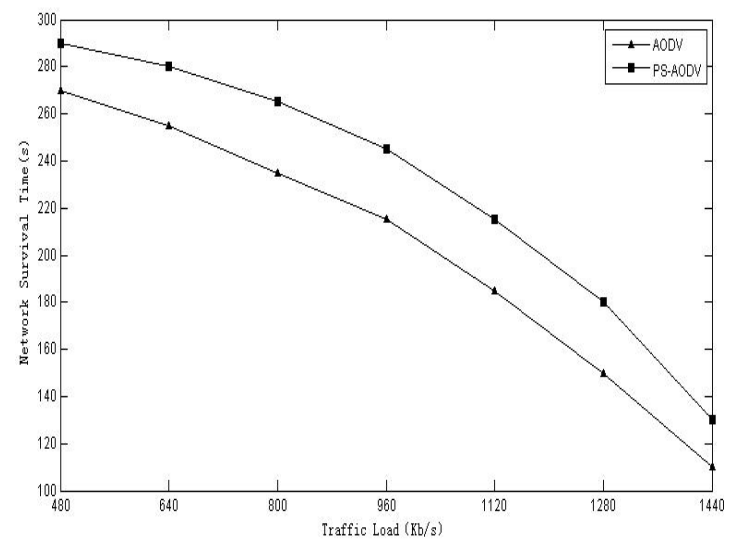

Figure 4. network survival time with different traffic load

Figure 4 shows that the network survival time of PS-AODV is longer than AODV. This is mainly due to PS-AODV node load is based on selection of smaller load, so that the energy consumption is much evenly to extend the network survival time.

\section{Conclusion And Perspectives}

In this paper, we propose a PS-AODV routing protocol based on an algorithm for path selecting and acknowledge refused, make improvements on AODV. According to the local node load and the RREQ packet metric value to determine whether response and make the path selection, in order to realize load balancing and to prevent excessive energy consumption of nodes in the center of the network. Simulation result shows that the PS-AODV protocol improves packet delivery ratio, reduces average end-to-end 
latency and decreases route overhead in high network load. In the future, we will try other routing protocols such as OLSR and GRP.

\section{REFERENCES}

[1] Eshghi F,Elhakeem A K.Performance analysis of ad hoc wireless LANs for real-time traffic[J].IEEE Journal on Selected Areas in Communications,2003,21(2):204-215.

[2] Chen Rong, Jiang Hong, Yang Huan. Comparative Analysis of Ad Hoc Networks Routing protocols[J].COMPUTER ENGINEER1NG AND DESIGN,2008,29(18): 4672-4675.

[3] Broch J.Maltz D.A.Johnson D.Hu Y.C.Jetcheva J A performance comparison of multi-hop wireless adhoc network routing rotocols 1998.

[4] University of Southern California The network simulator-Ns-2. 